\title{
ESTIMATIVA DO SEXO E IDADE POR MEIO DE MENSURAÇÕES CRANIANAS
}

\author{
Erasmo de Almeida Júnior*,Francisco Prado Reis**, Luis Carlos Cavalcante Galvão***, Hesmoney \\ Ramos de Santa Rosa ${ }^{* * * *}$, Naiá Costa*****
}

\begin{abstract}
Autor correspondente: Erasmo de Almeida Júnior. E-mail: anatjjunior@ig.com.br
*Professor da Disciplina de Anatomia Humana da Universidade Tiradentes - UNIT

**Professor Titular da Disciplina de Neuroanatomia da Universidade Tiradentes - UNIT

***Professor Titular do Departamento de Saúde da Universidade Estadual de Feira de Santana - UEFS

**** Professor de Clínica Neurológica da Faculdade de Medicina da Universidade Tiradentes- UNIT.

*****Acadêmica do Curso de Odontologia da União Metropolitana para o Desenvolvimento da Educação e Cultura (UNIME).
\end{abstract}

\begin{abstract}
Resumo
Introdução: A Odontologia Legal tem grande importância no processo de identificação, principalmente quando os profissionais da área recebem para análise apenas a porção cefálica do corpo. $O$ objetivo deste estudo é verificar o dimorfismo sexual e estimação da idade através de mensurações faciais. Material e Métodos: Os autores estudaram uma amostra de 150 crânios secos, sendo 80 masculinos e 70 femininos, que pertenceram a indivíduos com idade acima de 20 anos, e com sexo e idade conhecidos com absoluta segurança. Os crânios estudados eram de pessoas cujos familiares não reclamaram os ossos no tempo hábil administrativo estabelecido pelo Cemitério e que estavam sendo encaminhados para incineração. Foram realizadas as seguintes mensurações: curva frontal e distância Bregma/processo mastóide. Resultados: De acordo com a análise estatística, houve índice de acerto de $71,8 \%$ por regressão logística e $70 \%$ pela análise discriminante. Foi também verificada a média e intervalo de confiança através do teste t e utilizado o método da regressão linear múltipla para a predição da idade, constatando que o modelo não foi significativo $(p=0,5390)$. Conclusões: Os resultados permitiram a elaboração de metodologia estatística para o diagnóstico do sexo e idade em observações futuras.
\end{abstract}

Palavras-chave: Odontologia legal; Sexo; Idade; Crânio. 


\title{
SEX AND AGE ESTIMATION BY CRANIAL MEASUREMENTS
}

\begin{abstract}
Introduction: Forensic Dentistry has a great importance in the process of human identification, especially when the professional has only the cranium to analyze. The aim of this study is to verify the sex dimorphism and age estimation by the facial measurements. Materials and Methods: A sample of 150 dry skulls was studied, 80 male and 70 female, which belonged to individuals aged over 20 years old. The sex and age of the individuals were surely known. The skulls that have been studied belonged to people whose bones have not been claimed by their relatives in the determined time by the cemetery and were being sent for incineration. The following measurements have been done: frontal curve and distance between bregma and mastoid process. Results: According to the statistical analysis, there was success rate of $71.8 \%$ by logistic regression and $70 \%$ by the discriminant analysis. It was also verified the mean and confidence interval by $t$ test and used the method of multiple linear regression to predict age, evidencing the model was not significant $(p=0.5390)$. Conclusion: The results enable the development of a statistical methodology to identify gender and age in future observations.
\end{abstract}

Keywords: Forensic Dentistry; Sex; Age; Skull.

\section{INTRODUÇÃO}

O processo de identificação de pessoas, a partir de exames periciais, do segmento cefálico ou de partes dele, tem sido de significante importância para o esclarecimento de fatos de interesse jurídico-social. A tarefa exige técnicas e métodos mais precisos que venham auxiliar peritos médicos e odontólogos legistas a desempenharem eficientemente seu trabalho de auxiliar cientificamente a justiça quer no direito civil, penal, do trabalho e, em certos casos, até no direito administrativo.(1)

O capítulo da identificação, considerado uma das mais importantes funções do perito Médico e Odontolegal, é muito vasto e complexo, não podendo ser confundido com reconhecimento. Este último trata-se de um procedimento empírico baseado apenas em conhecimento prévio, cuja base de sustentação é unicamente testemunhal.(2)

De acordo com alguns autores, ${ }^{(3,4)}$ a investigação do crânio, em algumas situações, pode forne- cer elementos importantes para a identificação do sexo de uma pessoa. Essas situações podem se tratar de um indivíduo vivo, cadáver cronologicamente recente, cadáver em processo de putrefação ou de esqueletização, carbonizados, esqueleto completo ou partes dele, como por exemplo o crânio.

Segundo Kimmerle, Ross e Slice, ${ }^{(5)}$ a estimativa do sexo é um dos quatro pilares do protocolo antropológico, que consiste da análise métrica e avaliação visual das características do esqueleto, do crânio e da pelve. Desse modo, quanto mais mensurações e dados forem observados num laudo, mais confiável será o resultado.(2)

Qualitativamente, em geral, os crânios masculinos apresentam as estruturas mais grosseiras ou ásperas, devido às inserções musculares serem mais fortes. Dentre essas estruturas, podem ser citadas: a glabela, processos mastóides, rebordo 
orbitário, palato, abertura piriforme, extensão zigomática e rugosidades supraorbital. ${ }^{(6)}$

Quantitativamente, os crânios masculinos são também, em geral, maiores do que os femininos, podendo-se relatar algumas características como: capacidade craniana, peso, diâmetro ântero-posterior, diâmetro transverso, altura craniana, largura bizigomática e espessura óssea. ${ }^{(7,8)}$ Segundo alguns autores $^{(9)}$ o sexo do indivíduo pode ser facilmente estimado com o uso de um pequeno conjunto de 11 variáveis craniométricas.

O exame do dimorfismo sexual através do crânio tem sido objeto de numerosos estudos morfológicos e craniométricos, mas a desvantagem desses estudos é que eles são dependentes da experiência do operador. $\mathrm{Na}$ verdade, o grande problema que envolve a Antropologia Forense é a variabilidade morfológica e métrica que ocorre em populações diferentes, devido a fatores climáticos, alimentares e sócio-organizacionais, sendo necessária a realização de mais estudos em nossa população. ${ }^{(10)}$

O presente estudo é uma tentativa de verificar o dimorfismo sexual e estimar a idade, examinando crânios secos através das seguintes medidas: curva frontal e distância Bregma/processo mastóide do lado direito.

\section{MATERIAL E MÉTODO}

Foram utilizados para o estudo 150 crânios secos, 70 do sexo feminino e 80 masculinos, todos maiores de 20 anos de idade. Os crânios foram obtidos de acordo com a lei $n^{\circ} 8501$ de 1992, que trata do uso de cadáveres não reclamados para uso em estudos e pesquisas. Estes crânios pertencem ao acervo do laboratório de Anatomia Humana da Universidade Tiradentes (UNIT), localizada na cidade de Aracaju, Estado de Sergipe, Brasil, local onde também foram realizadas as mensurações dos mesmos. Foram incluídos na pesquisa os crânios que não apresentavam qualquer tipo de dano, ou variações anatômicas relacionadas à região de estudo. Não foram levados em consideração dados socioeconômico e cor da pele.
Para a obtenção das medidas foi utilizado um compasso de espessura da marca Vonder e uma fita métrica graduada em milímetros devidamente calibrada. Foram tomadas medidas lineares que correspondiam às distâncias entre os seguintes pontos: Násio ao Bregma (Násio é o ponto localizado na união da sutura frontonasal com a sutura internasal, e o Bregma que é o ponto localizado na união das suturas coronária e sagital, esta distância corresponde à curva frontal - $d n b$ ); e distância do Bregma ao processo mastóide do lado direito (utilizou-se o ponto mais inferior do processo mastóide - dbpm). A amostra foi estatisticamente tratada utilizando-se os seguintes métodos: a princípio foi utilizado o teste $t$, que objetiva testar a hipótese de que as médias das medidas do sexo masculino difere das médias das mesmas medidas do sexo feminino, sendo assim, para cada variável se fez um teste para a hipótese de que as médias das medidas do sexo masculino difere das médias das medidas do sexo feminino. Através deste mesmo teste se verificou o desvio padrão e o intervalo de confiança. Uma vez avaliada a existência de diferenças entre as médias das variáveis entre o sexo masculino e o feminino, um modelo de análise de regressão logística permitirá o desenvolvimento de um método para predizer a probabilidade de pertinência de um crânio pertencer a um dos sexos, mais especificamente nesse caso, ao sexo feminino. Outro método utilizado foi o da análise discriminante, que é uma alternativa à regressão logística e foi aplicada para se comparar os resultados. A técnica se baseia no desenvolvimento de um conjunto de funções lineares discriminantes, mais especificamente, uma para cada possibilidade de predição, ou seja, uma para o sexo masculino e outra para o sexo feminino. O último método utilizado foi o da regressão linear, específico para a predição da idade que começa por uma seleção de variáveis e que permite a construção de uma equação para predição da idade. Em todos os testes utilizados foi adotado um nível de significância de $5 \%$ e os cálculos foram realizados com o uso do sistema SAS. 


\section{RESULTADOS}

Os resultados obtidos na pesquisa de campo foram agrupados e submetidos à análise estatística, apresentando os seguintes resultados: a) Caracterização da amostra

A amostra foi caracterizada com base na comparação das médias das variáveis estudadas de cada sexo, cujos valor médio, desvio padrão e limites de intervalos de confiança estão representados na Tabela 1.

Tabela 1 - Estatísticas básicas (média, desvio padrão e limites dos intervalos de confiança da média - 95\%) das variáveis observadas ( $n: 150$ ) e teste $t$ para comparação das médias das variáveis dnb e dbpm nos diferentes sexos

LIMITE DO INTERVALO DE CONFIANÇA DA MÉDIA (95\%)

\begin{tabular}{|c|c|c|c|c|c|}
\hline VARIÁVEL & SeXo & MÉDIA(MM) & $\begin{array}{l}\text { DESVIO } \\
\text { PADRÃo }\end{array}$ & SUPERIOR & INFERIOR \\
\hline$d n b$ & Feminino & 126,086 & 7,851 & 127,958 & 124,214 \\
\hline valor-p: 0,1548 & Masculino & 127,825 & 7,044 & 129,393 & 126,257 \\
\hline dbpm & Feminino & 149,400 & 6,464 & 150,941 & 147,859 \\
\hline valor-p: <0,0001 & Masculino & 155,175 & 6,665 & 156,658 & 153,692 \\
\hline
\end{tabular}

Fonte: elaboração dos autores.

A análise de variância dos dados mostrou que os indivíduos do sexo masculino apresentaram médias das variáveis maiores que as do sexo feminino. De acordo com o teste $t$, ocorreu diferença significativa das médias da variável $d b p m$, não ocorrendo o mesmo na variável $d n b$. Os intervalos de confiança da variável dbpm foram desconexos, ou seja, não houve interposição de faixas, indicando que esta variável apresenta bons indícios para discriminação do sexo a partir das medidas realizadas. Com relação a variável $d n b$, houve uma pequena interposição de faixa, o que não prejudicou em nada a aplicação de outros métodos estatísticos mais específicos.

b) Análise de Função Discriminante

Para a análise discriminante, foram construídas duas equações a partir dos dados encontrados, sendo que o resultado maior entre as duas equações corresponde ao sexo indicado:

Feminino $=-288,71954+1,16616 x d n b+2,88088 x$ dbpm

Masculino $=-307,78513+1,14096 x d n b+3,02708 x$ dbpm

A avaliação da capacidade de discriminação foi obtida através de um processo de ressubstituição ou reclassificação no qual os dados conduziram a uma estimativa do sexo. Por este processo, houve índice de acerto da ordem de $70 \%$ em ambos os sexos, apresentando uma taxa total de erros de $30 \%$, inferior àquela obtida em dependência do acaso, que seria de 50\% (Tabela 2). 
Tabela 2 - Ressubstituição contrapondo os sexos observados e preditos através das funções lineares discriminantes a partir dos dados que foram usados na estimativa dos parâmetros do modelo (n: 150)

\begin{tabular}{|c|c|c|c|}
\hline \multirow[b]{2}{*}{ SEXO OBSERVADO } & \multicolumn{2}{|c|}{ SEXO PREDITO } & \multirow[b]{2}{*}{ TOTAL } \\
\hline & FEMININO & MASCULINO & \\
\hline \multirow{2}{*}{ Feminino } & 49 & 21 & 70 \\
\hline & 70,0 & 30,0 & 100,00 \\
\hline \multirow{2}{*}{ Masculino } & 24 & 56 & 80 \\
\hline & 30,0 & 70,0 & 100,00 \\
\hline \multirow{2}{*}{ Total } & 73 & 77 & 150 \\
\hline & 48,67 & 51,33 & 100,00 \\
\hline Porcentagem de erros & 30,0 & 30,0 & 30,0 \\
\hline Acertos ao acaso & 50,0 & 50,0 & \\
\hline
\end{tabular}

Fonte: elaboração dos autores.

c) Regressão logística

$\mathrm{Na}$ regressão logística, partindo do estudo de seleção das variáveis através do método Stepwise, foi verificado que para apoiar a decisão de seleção do sexo seria adequado o uso de uma das duas variáveis medidas: a distância dbpm.

Através dos dados encontrados, foi elaborada a seguinte equação:

$$
\text { Logito }=20,1855-0,1335 \times \mathrm{dbpm}
$$

Este modelo foi considerado altamente significativo e permitiu estimar a pertinência de uma medida pertencente ao sexo feminino. A transformação deste logito na probabilidade de pertinência da medida à pessoas do sexo feminino foi obtida através da seguinte função:

$$
\begin{aligned}
& \text { pf }=e^{20,1855-0,1335 \times d b p m} /(1+e)^{20,1855-0,1335 \times d b p m} \\
& \text { onde: } e=2,71828
\end{aligned}
$$

Através desta equação, foi atingido um índice de concordância de $71,8 \%$. Os parâmetros de cor-relação indicaram valores em torno de $48 \%$ ( $\mathrm{Ta}$ bela 3).

Tabela 3 - Associação entre probabilidades estimadas e respostas observadas

\begin{tabular}{lccc}
\hline Porcentagem de concordância: & 71,8 & D de Somer : & 47,0 \\
Porcentagem de discordância: & 24,8 & Gamma : & 48,7 \\
Porcentagem de empate: & 3,5 & Tau-a: & 0,236 \\
Pares : & 5600 & c : & 0,735 \\
\hline
\end{tabular}

Fonte: elaboração dos autores. 
d) Regressão Linear Múltipla

A estimativa da idade foi realizada através de um modelo de regressão linear múltipla, que foi inicia- do através da análise de variância do modelo de regressão. De acordo com os dados, foi constatado que o modelo de predição da idade não foi significativo ( $p$ : 0,5390 ), demonstrado na Tabela 4 .

Tabela 4 - Análise de variância do modelo de regressão linear múltipla para predição das idades em função das variáveis

\begin{tabular}{|c|c|c|c|c|c|}
\hline CAUSA DE VARIAÇÃO & GL & $\begin{array}{c}\text { SOMA DE } \\
\text { QUADRADOS }\end{array}$ & $\begin{array}{c}\text { QUADRADOS } \\
\text { MÉDIOS }\end{array}$ & VALOR F & VALOR-P \\
\hline Modelo & 1 & 110,4010 & 110,4010 & 0,38 & 0,5390 \\
\hline Resíduo & 148 & 43093 & 291,17134 & & \\
\hline Total corrigido & 149 & 43204 & & & \\
\hline
\end{tabular}

Fonte: elaboração dos autores.

\section{DISCUSSÃO}

Excetuando-se a pelve, o crânio é amplamente considerado o melhor indicador para o diagnóstico do sexo. ${ }^{(11)} \mathrm{Na}$ grande maioria dos estudos a respeito da identificação do sexo de um indivíduo, a partir do exame da porção cefálica, tem sido utilizado o método qualitativo. São frequentemente estudadas características tais como: os seios frontais, dentes, glabela, espessura dos ossos dos arcos superciliares, forma do mento, aspecto da superfície óssea devido à ação da musculatura, processos mastóides, eminências parietais, fossa canina, arcos alveolares, processos coronóides e articulação frontonasal. ${ }^{(1,6)}$

Em alguns estudos são utilizadas medidas entre pontos pré-estabelecidos, que proporcionam critérios mais seguros na questão do diagnóstico do sexo. Muitos autores têm contribuído de maneira clara e científica para a determinação do sexo através de métodos quantitativos. ${ }^{(12,13,14,15)}$ Essa literatura, na sua maioria, utilizou amostras estrangeiras, fato que tem limitado sua aplicabilidade à população brasileira.

Com relação a mandíbula, é elevado o grau de dimorfismo sexual, especialmente à nível do seu ramo, que normalmente, devido ao processo de mastigação, é submetido a maior estresse do que qualquer outro osso do crânio. ${ }^{(16)}$ No presente estudo, as medidas das distâncias Násio/Bregma $(d n b)$ e Bregma/processo mastóide do lado direito $(\mathrm{dbpm})$ comparadas ao que se depreende entre os métodos qualitativos, pareceram mais eficientes, visto terem fornecido valores matemáticos que, analisados estatisticamente, podem contribuir para a redução ou a eliminação de erros de observação, interpretação pessoal e deformação anatômica relativa. Os testes estatísticos utilizados no presente estudo, por exemplo, forneceu resultados satisfatórios. Alguns autores também obtiveram bons resultados com relação a estes métodos. $(12,13,14,15)$

A regressão logística tem sido empregada também em estudos semelhantes por outros autores. $(2,17)$ Através de uma seleção de variáveis identificadas pelo método Stepwise, foi constatado que seria adequado o uso de uma dentre as duas variáveis medidas para apoiar a decisão de seleção do sexo: dbpm. A partir deste método, obtemos um índice de concordância de $71,8 \%$. Alguns autores, utilizando o mesmo tratamento estatístico, chegaram também a resultados considerados satisfatórios: por exemplo, Galvão(2) obteve índices de acerto de $92,9 \%$ e $94,7 \%$ para os sexos feminino e masculino, respectivamente. 
Quanto à análise discriminante empregada também por alguns autores, ${ }^{(14,15,18)}$ no presente estudo foi encontrado um índice de acerto de 70,0\% em dados do sexo feminino e 70,0\% para dados masculinos, com uma taxa total de erro de $30,0 \%$, sendo inferior àquela obtida ao acaso, que seria de $50 \%$. Com relação a predição da idade, em que foi utilizado o método da regressão linear múltipla, o modelo não foi considerado significativo, apresentando um valor de $p=0,5390$ muito maior que o nível de significância de 0,05 .

Os dados obtidos no presente estudo permitiram concluir que a medida das variáveis estudadas foram menores em crânios do sexo feminino. Este resultado está de acordo com alguns autores, que em seus estudos avaliaram áreas faciais quantitativamente. ${ }^{(19,20,21)} \mathrm{A}$ utilização de diferentes métodos estatísticos teve o objetivo de diminuir a margem de erro. A utilização desses métodos ofereceu maior confiabilidade nos resultados, quando da aplicação nos casos de identificação, podendo ser utilizados em serviços de Antropologia Forense e nos Institutos Médicos Legais. Considerando que o presente estudo utilizou uma amostra nacional, pode ser admitido que isto venha evitar alguns dos inconvenientes apresentados pelas amostras estrangeiras resultante de fatores tais como: clima, alimentação, condição socioeconômica e qualidade de vida, que poderão possivelmente interferir no diagnóstico.

\section{CONCLUSÃO}

A metodologia realizada para a estimativa do sexo através de medida de distâncias entre pontos da face e do crânio, de acordo com os resultados da técnica da regressão logística, com a amostra utilizada, obteve-se índice de acerto de $71,8 \%$. A amostra foi também, durante a investigação, submetida à análise de função discriminante, por média e pelo intervalo de confiança. Quanto à estimativa da idade, o modelo do estudo não apresentou significância estatística, não demonstrando, portanto, confiabilidade. É possível que metodologias como a empregada no presente estudo, isolada ou ao lado de outras, possam vir a contribuir para o acerto de pesquisas sobre a investigação do sexo dentro da Medicina Legal e de outros campos de estudo afins.

\section{REFERÊNCIAS}

1. França GV. Medicina Legal. $5^{a}$ ed. Rio de Janeiro: Guanabara Koogan; 1998.

2. Galvão LCC. Determinação do sexo através da curva frontal e apófise mastoide [tese]. Piracicaba: Faculdade de Odontologia de Piracicaba; 1998.

3. Francesquini Junior L, Francesquini LA, De La Cruz BM, Prereira SD, Ambrosano GM, Barbosa $C M$ et al. Identification of sex using cranial base measurements. J. forensic odontostomatol. 2007;25(1):7-11.

4. Patil KR, Mody RN. Determination of sex by discriminant function analysis and stature by regression analysis: a lateral cephalometric study. Forensic sci. int. 2005; 147(2-3): 175-80.

5. Kimmerle EH, Ross A, Slice D. Sexual dimorphism in America: geometric morphometric analysis of the craniofacial region. Forensic sci. int. 2008; 53(1): 54-7.

6. Rogers TL. Determing the sex of human remains through cranial morphology. J. forensic sci. 2005; 50(3):493-500.

7. Gapert R, Black S, Last J. Sex determination from the foramen magnum: discriminant function analysis in an eighteenth and nineteenth century British sample. Int. j. legal med. 2009;123(1): 25-33.

8. Hatipoglu HG, Ozcan HN, Hatipoglu US, Yuksel E. Age, sex and mass index in relation to calvarial diploe thickness and craniometric data on MRI. Forensic sci. int. 2008; 182(1-3): 46-51.

9. Konigsberg IW, Algee-Hewitt BF, Steadman DW. Estimation and evidence in forensic anthropology: sex and race. Am. j. phys. anthropol. 2009;139(1):77-90.

1O. Veyre-Goulet SA, Mercier C, Robin O, Guerin C. Recent human sexual dimorphism study using 
cephalometric plots on lateral teleradiography and discriminant function analysis. J. forensic sci. 2008; 53(4): 786-9.

11. Spradley MK, Jantz RL. Sex estimation in forensic anthropology: skull versus poscranial elements. J. forensic sci. 2011;56(2):289-96.

12. Osipov B, Harvati K, Nathena D, Spanakis K, Karantanas A, Kranioti EF. Sexual dimorphism of the bony labyrinth: a new age-independent method. Am. j. phys. anthropol. 2013; 151(2): 290-301.

13. Nidugala $\mathrm{H}$, Bhargavi $\mathrm{C}$, Avadhani R, Bhaskar B. Sexual dimorphism of the craniofacial region in a South Indian population. Singap. med. j. 2013;54(8):458-62.

14. Jiménez-Arenas JM, Esquivel JA. Comparing two methods of univariate discriminant analysis for sex discrimination in an lberian population. Forensic sci. int. 2013; 228(1-3): 175.el-4.

15. Ogawa Y, Imaizumi K, Miyasaka S, Yoshino M. Discriminant functions for sex estimation of modern Japanese skulls. J. forensic legal med. 2O13;2O(4): 234-8.

16. Saini V, Srivastava R, Rai RK, Shamal SN, Singh TB, Tripathi SK. Mandibular ramus: an indicator for sex in fragmentary mandible. J. forensic sci. 2011;56 Suppl 1:13-6.

17. Ikeda T, Nakamurs M, Itoh M. Sex differences in the zygomatic angle in japanese patients analyzed by MRI. Aesthet. plast. surg. 1999;23(5):349-53.

18. Gonzalez RA. Determination of sex from juvenile crania by means of discriminant function analysis. J. forensic sci. 2012; 57(1): 24-34.

19. Galdames IS, Zavando D.Age effect in the morphological traits performance for sex determination in human skulls and mandibles. Int. j. morphol. 2012;30(1):296-301.

2O. Almeida Jr E, Galvão LCC, Flores PSC, Araújo TM. Investigação do sexo através de uma área triangular facial formada pela interseção dos pontos: forame infraorbital direito, esquerdo e o próstio, em crânios secos de adultos. Rev. ciênc. méd. biol. 2010; 9(1): 8-12.

21. Franklin D, Cardini A, Flavel A, Kuliukas A. Estimation of sex from cranial measurements in a Western Australian population. Forensic sci. int. 2013;229(1-3):158.el-8. 